\title{
THE IMPORTANCE OF URINE CULTURES IN THE FOLLOW-UP AFTER FIRST FEBRILE UTI
}

\author{
Albert Lama ${ }^{1}$, Diamant Shtiza ${ }^{2 *}$, Enkelejda Shkurti $^{3}$ \\ ${ }^{1}$ University Hospital Centre "Mother Tereza" Tirana, Department of Pediatrics, \\ Service of Emergency, Tirana, Albania; \\ ${ }^{2 *}$ University Hospital Centre "Mother Tereza" Tirana, Department of Pediatrics, \\ Service of Nephrology-Dialysis, Tirana, Albania; \\ ${ }^{3}$ University of Medicine, Faculty of Technical Medical Sciences, Tirana, Albania; \\ *Corresponding Author Diamant Shtiza, e-mail: diamantshtiza@yahoo.com;
}

Received November 2021; Accepted December 2021; Published January 2022;

DOI: $\underline{\text { https://doi.org/10.31407/ijees12.112 }}$

\begin{abstract}
Background: Studies evaluating the role and utility of the routine follow-up of urine cultures after the first febrile urinary tract infection are limited. Was evaluated the validity of routine follow-up urine cultures in a group of children who had their first episode of urinary tract infection at the age_of 1month to 6 years. Study design: The research findings are derived from one prospective randomised study; 676 (468 girls) children aged of 1 month to 6 years were included in the study. The time of recurrence and the possible symptoms during the recurrent urinary tract infection (UTI) were also investigated. The follow-up period after the first UTI was of 1 year. Urinalysis and urine culture follow-up were performed every month during the first 6 months and then every other month. The urine was collected by sterile bag collection in all children. Results: were performed 4796 routine urinalysis and urine cultures were obtained; 158 patients $(23 \%)$ had a positive urine culture during the follow-up, $106(15,6 \%)$ of them were girls. $54(1,1 \%)$ febrile UTI were excluded; $4504(95 \%)$ urine cultures were negative; $68(1,4 \%)$ contaminations; $114(2,4 \%)$ asymptomatic bacteriuria; $56(1,2 \%)$ lower UTI. E. Coli was most frequent microorganism; it was found in $112(50 \%)$ of all cases and in 77\% of upper UTI. The cost of urinalysis was 2,3 euro, that of urine culture was 11,6 euro, that of urine bag was 1,2 euro and that of urine cup 0,9 euro: a total of 16 euro per patient for 1 control. The total cost of 4796 controls was 76.376 euro. Conclusions: We noted a high negative predictive value, which authenticates that a negative urinalysis is followed almost by a negative urine culture. Urine culture follow-up is not necessary for the children after first febrile UTI. Such an approach would result in significant cost savings.
\end{abstract}

Keywords: urine culture follow-up, febrile UTI 